\title{
Fish Bone-related Intra-abdominal Abscess Treated With Antibiotic Therapy
}

\author{
Yusaku Kajihara®
}

Department of Gastroenterology, Fuyokai Murakami Hospital, Aomori, Japan

A 72-year-old Japanese male patient presented to the gastroenterology department with a 2-week history of right lower quadrant (RLQ) abdominal pain. He had well-controlled type 2 diabetes mellitus (hemoglobin A1c of 6.2\%). Vital signs were normal. His abdomen was soft with mild tenderness in the RLQ. Laboratory evaluation showed elevated a leucocyte count and a serum C-reactive protein (CRP) level at 13,100 cells/ $\mu \mathrm{L}$ (normal range 3500-8500) and $14.31 \mathrm{mg} / \mathrm{dL}$ (normal value $<0.30$ ), respectively. Contrast-enhanced computed tomography (CT) revealed a small amount of ascites and an 84 x $66 \mathrm{~mm}$ mass containing a linear opacity in the left upper abdominal cavity (Figure 1). No evidence was observed for free intra-abdominal air. Esophagogastroduodenoscopy and colonoscopy showed no abnormality. Serum tumor markers, including carcinoembryonic antigen, carbohydrate antigen 19-9, DUPAN-2, and Span-1,

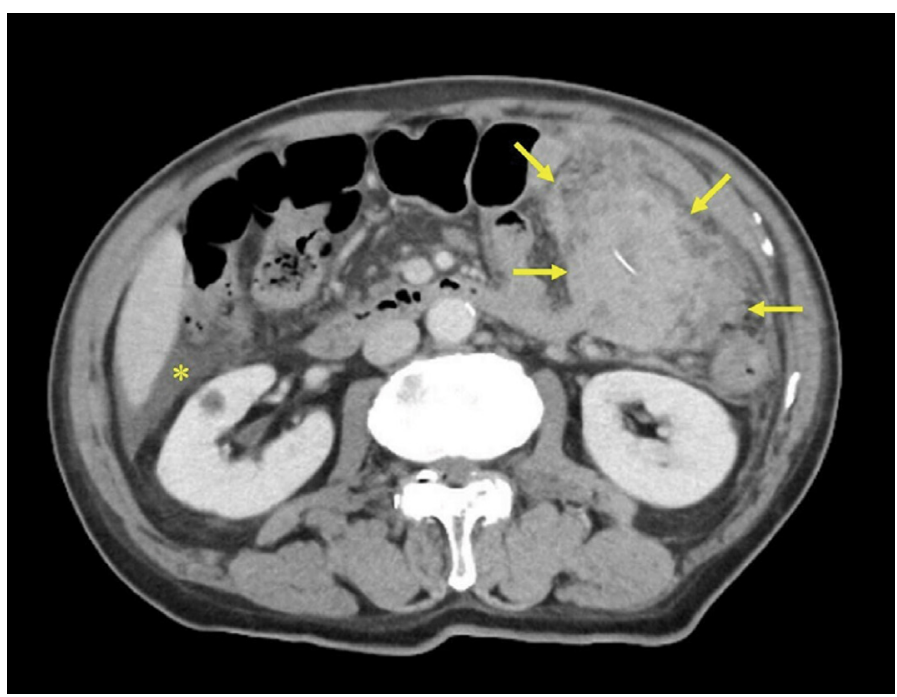

FIG. 1. Contrast-enhanced computed tomography image showing a small amount of ascites (asterisk) and an $84 \times 66 \mathrm{~mm}$ mass containing a linear opacity in the left upper abdominal cavity (arrows). were within the normal range. The patient denied any history of fish bone ingestion. However, his family reported that he had eaten cod baked in foil several days before the presentation. Therefore, a diagnosis of the fish bone-related intra-abdominal abscess was made. The patient's condition was stable, thus parenteral nutrition and antibiotic therapy were administered. The abdominal pain disappeared within 2 days after the initiation of intravenous sulbactam/cefoperazone (SBT/CPZ) (1 g twice daily). The leucocyte counts and serum CRP levels also decreased immediately. Oral intake was restarted 7 days later, and oral levofloxacin (500 mg once daily) was prescribed as an alternative to SBT/CPZ. At the 1-month follow-up, CT confirmed the resolution of ascites and the intra-abdominal abscess despite the retained fish bone that measures approximately $2 \mathrm{~cm}$ (Figure 2); thus, the antibiotic therapy was completed.

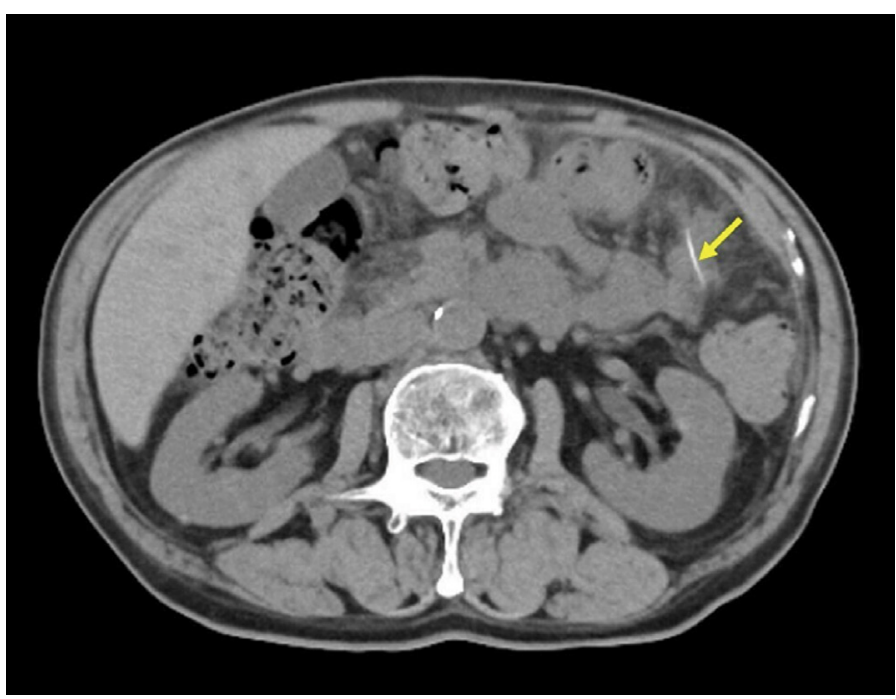

FIG. 2. Follow-up computed tomography image showing the resolution of ascites and the intra-abdominal abscess despite the retention of the fish bone, measuring approximately $2 \mathrm{~cm}$ (arrow).

Corresponding author: Yusaku Kajihara, Department of Gastroenterology, Fuyokai Murakami Hospital, Aomori, Japan

e-mail: y kaji2012@yahoo.co.jp

Received: October 16, 2021 Accepted: December 20, 2021 Available Online Date: March, 14, 2022 • DOI: 10.4274/balkanmedj.galenos.2021.2021-10-64

Available at www.balkanmedicaljournal.org

ORCID iDs of the authors: Y.K. 0000-0002-3233-8829.

Cite this article as:

Kajihara Y. Fish Bone-related Intra-abdominal Abscess Treated With Antibiotic Therapy. Balkan Med J.; 2022; 39(2):157-8

Copyright@Author(s) - Available online at http://balkanmedicaljournal.org/ 
Accidental foreign body ingestion is common in clinical practice. The majority of the ingested foreign bodies pass through the gastrointestinal (GI) tract without any complications. ${ }^{1}$ Fish bones are the most common objects that cause bowel perforation due to their sharpness. ${ }^{2,3}$ Intra-abdominal abscess can result from bowel perforation. ${ }^{2}$ The main symptoms include acute or chronic abdominal pain and fever, which are non-specific. ${ }^{2,4}$ Obtaining a history of fish bone ingestion is difficult since most patients tend to forget the ingestion of fish bones. ${ }^{3,5}$ Fish bones are usually invisible on radiography due to their radiopacity. ${ }^{3}$ Fish bone perforation typically appears on $\mathrm{CT}$ as a linear calcified lesion surrounded by an area of inflammation. ${ }^{3} \mathrm{CT}$ is useful in diagnosing fish bone perforation of the GI tract; however, the accuracy of CT is limited due to insufficient observer awareness. ${ }^{3}$ An intra-abdominal abscess secondary to bowel perforation commonly requires surgical intervention, such as drainage and foreign body removal. ${ }^{2}$

In the present case, abdominal pain in the left upper quadrant was not observed. The patient's chief complaint was considered as a referred pain. Moreover, a large abscess had completely healed by antibiotic therapy without surgical intervention. Physicians should be cautious of a recurrent abscess due to the retained fish bone, and conservative treatment may be suitable for patients with mild symptoms.

Patient Consent for Publication: Written informed consent was obtained from the patients.

Conflict of Interest: No conflict of interest was declared by the author.

\section{REFERENCES}

1. Hakeem A, Shanmugam V, Badrinath K, Dube M, Panto P. Delay in diagnosis and lessons learnt from a case of abdominal wall abscess caused by fishbone perforation. Ann R Coll Surg Engl. 2015;97:e39-42. [Crossref]

2. Chen CK, Su YJ, Lai YC, Cheng HK, Chang WH. Fish bone-related intra-abdominal abscess in an elderly patient. Int J Infect Dis. 2010;14:e171-172. [Crossref]

3. Goh BK, Tan YM, Lin SE, et al. CT in the preoperative diagnosis of fish bone perforation of the gastrointestinal tract. Am J Roentgenol. 2006;187:710-714. [Crossref]

4. Choi Y, Kim G, Shim C, Kim D, Kim D. Peritonitis with small bowel perforation caused by a fish bone in a healthy patient. World J Gastroenterol. 2014;20:1626-1629.

5. Gupta K, Madhavan SM, Augustine AJ. Ingested fishbone causing pericolic abscess. ACG Case Rep J. 2017;4:e28. [Crossref] 